\title{
Application of Clothing Ergonomics in Fashion Design
}

\author{
Xiaoyan Yang \\ Huanghe Science and Technology College \\ Zhengzhou, Henan, China 450000
}

\begin{abstract}
Clothing ergonomics is a new comprehensive subject, although the history of the development of this discipline is not long, the speed of development is very fast. This paper mainly expounds the clothing ergonomics is the system engineering discipline with "human centered", clothing as the media, and environment as condition. It combines professional clothing characteristics and ergonomics content to constitute an independent system, and the purpose is to completely change the traditional concept of the past, realizes harmony and unity of human and the clothing, humane and the environment, "beauty in form" and "efficiency" in the process of the clothing designing, so that clothing art and wearing actual effect can reach the best matching condition of comfort, health, safety, health, function, appearance, personality.
\end{abstract}

Keywords-ergonomics; clothing; design; application

\section{INTRODUCTION}

The design of clothing ergonomics gives people a scientific and systematic guidance in line with the scientific data and test data, and provides people design concept accord with their own needs. In the process of clothing creation, human, clothing and environment interrelate and influence each other and constitute a system, called "the human - clothing environment" system, the "clothing" of the system refers to all dresses that people wear, dominating all dress content, the environment is the external environment condition that affect the people and clothing. "Human - clothing - environment" system is the basis of the clothing design, and the clothing design must reflect the value of the system, that is to make people be comfortable, sanitary and beautiful in the clothing behavior in the system.

\section{The MEANING OF ClOTHING ERGONOMICS}

Clothing ergonomics is the frontier subject of fashion design, which is a branch of human engineering. The reason of that the clothing ergonomics is a branch of human engineering is because its system purpose, value, function are consistent with each other, and clothing more specifically serves as a carrier of human body engineering, and become a medium of discipline theory and the practice.

The clothing ergonomics is the system engineering discipline with "human centered", clothing as the media, and environment as condition in essence. It mainly studies the relevant problems between clothing and people, clothing and environment, so that they can achieve harmony and unity.
Clothing ergonomics can also say it is a kind of applied science that improves the whole function of clothing, which bases on the characteristics of human body form and motor function and full considers the harmony and comfort of human body and clothing. It brings together the clothing materialogy, textile science, human psychology, human anatomy, environmental hygiene, anthropometry, fashion design, medical science and many other disciplines.

Clothing ergonomics combines with the characteristics of the garment specialty and the content of human engineering and constitutes an independent system. Its purpose is to make "clothing adapts to people", completely change the traditional concept of the past, "people adapts to clothes"; realize harmony and unity of human and the clothing, humane and the environment, "beauty in form" and " efficiency " in the process of the clothing designing; make the each index of clothing medium and the various requirements of human body adopt to each other; make the clothing design as far as possible to suit the needs of the human body; make the clothing art and wearing actual effect reach the best matching condition of comfort, health, safety, health, function, appearance, personality.

\section{The Position OF Clothing ERgOnOMics IN FASHION DESIGN}

The design concept of human engineering is essential for all mechanical design, and the design concept of clothing ergonomics is essential for the clothing design. Previous costume design focuses on "beauty in form", in order to show a kind of art style to pursue fashion and pop, strong subjective desire, less thinking of the factors of person, so results in the designed works and clothing can only be appreciated, and is not easy to be understood. By the end of 1990s, the "people oriented" and "humanized design" has become the focus of the design industry, especially in the clothing industry. When the designers design clothing, the prerequisite of "beauty in form" is that the clothing structure design is scientific, reasonable, and helpful to the movement of the body; pay attention to the skin to meet the health requirements, in accordance with the human physiological and psychological indexes; pay attention to clothing material accord with the requirements of the human body, is easy for live and maintenance, to realize the true sense of the "clothes adapt to people".

For example, in the process of designing swimwear, according to the design idea of clothing ergonomics, 
swimwear should be designed to be convenient for the athlete to violently swim, otherwise it will make athletes be limited, and greatly affect the performance.

And another example, in the process of designing dresses, in order to show the characteristic of the female body, designers often use a belt to tie in the waist, and the female body characteristics show out. But they ignored that the clothing emphasizes the ventilation and heat dissipation in summer, tying a belt in waist hinders ventilation. Only modify the design in accordance with the clothing ergonomics design, this problem can be solved. Pleating instead of tying a belt in the waist realizes the intention of waisting. This not only shows the characteristic of the female form, but also in line with the requirements of health and hygiene, and good ventilation performance will enhance the wearer comfort. Through the above cases, clothing ergonomics plays a very important role in fashion design.

\section{APPLICATION OF CLOTHING ERGONOMICS IN FASHION DESIGN}

The aim of the study of clothing ergonomics is to make the harmonious match between the clothing design and the human and the environment, the pursuit is the best performance, and the requirements are the subjects that it will relate to the subjects: Clothing design science, hygiene, anthropometry, physiology, psychology, human anatomy, medical and other subjects are combined to the concept of fashion design, and make the designed clothing be more scientific, reasonable, efficient, finally, reach the best matching state of the system of "human-clothing - environment". The following example illustrates the application of clothing ergonomics in the clothing design.

\section{A. Application of Each Parts of Human Body in Fashion Design}

Clothing ergonomics requires clothing design and human body parts to adapt to each other, make the human body and clothing interface an overall harmony of internal and external and synchronization, shows the groove and optimal performance.

Clothing design has a unique perspective on anthroposcopy, unlike the tool design that the human hand, joint shape is the main; Also unlike human engineering in terms of furniture design, the main is the human physiological function, spine form, the scope of activities; and the costume designer uses person's trunk and limbs as the center to fully show the harmonious beauty and the optimum efficiency of the human body and clothing.

In order to the clothing design can be carried out smoothly, according to the different parts of the body playing different roles, the designers and design groups divide the human body into four areas, namely the fitting area, free area, function area and design area. Fitting area is the basic support for clothing design; the free zone and the function area are the important parts to meet the human body's movement function in the fashion design; the design area is the free change of clothing space, rich modelling area, is the part that reflects designers' design philosophy and the design style. Experts say: "in the modern fashion design, clothing space should extrude function, fit, free activities, trying to display the possibility of human activities, does not inhibit and restrict the range of activities, comply with the process of human life, realizes the translation from the 'people adopts to clothes' to 'clothing services people'".

\section{B. The Application of Neck, Shoulder, Chest, Waist, Limbs, Buttocks and Other Parts in the Clothing Design}

In process of costume designing, clothing should adopt to neck, shoulder, chest, waist, limbs, buttocks and other parts of body to show the best aesthetic feeling.

- Designing clothing collar is based on the structural form around the neck and suture, and if not considering the structural characteristics of the neck, the costume will be very odd.

- The shoulder plays a very important role in the garment modeling, because it has a big influence on people's gender, body type, especially the style of clothing. Whether clothing structure fits shoulder directly affects the appearance of clothing, people's comfort and the activities of the upper limb. Therefore, in the process of clothing designing to the key parts, such as neck, shoulder, should be done strictly in accordance with the requirements of the clothing ergonomics.

- Chest occupies a special position in clothing design, it is the key part of the women clothing design, the success or failure of the design of this part is related to the overall formal beauty, also play a key role whether the human body trunk is comfortable, health, heart and lung activity is normal. Therefore, it is very important to study the relationship between the appearance of chest and the garment modeling in the process of designing clothing.

- Clothing Ergonomics requires that the waist baseline as a standard, when people design trousers and skirts, and coordinate with other forms. And remind designers to pay special attention to whether the form of trouser gluteal fold fit the person, if not, pants are too tight or too loose, and it is also uncomfortable and ugly.

- The most flexible part of the human body is the upper limb. In the process of designing clothing, sleeve type must match the upper limb structure and movement, especially the armpit part can not be thick. Because the arm is closed to the body when it have a rest, if the arms stay braced status, it will increase the discomfort and fatigue, also does not conform to the hygiene's requirements.

\section{The Application of Anthropometry in Garment Design}

Anthropometry is an important basic subject in garment ergonomics, and human form and size measurement are the important parts of clothing ergonomics. The data of human body measurement plays an important role in many industries, especially in clothing industry. Originating from the comfort, fit, function and other the engineering requirements of 
improving functions of human body, it is necessary that the exact human parameters as the reference resources for the clothing design, so that the human body and clothing achieve a reasonable match.

\section{Application of Clothing Materials in Fashion Design}

In the design of clothing, clothing material is one of the three elements of clothing, which is the material basis of clothing. Its great varieties and the different performance characteristics play the different role in human body. Clothing ergonomics research holds that natural fibers cotton, wool, silk and chemical fiber polyester and polypropylene fiber have the main functions and characteristics of the clothing material. In particular, it can reflect the comfortable, soft, elastic and sanitary properties of clothing materials, and it is the first choice for the clothing design in line with the requirements of human engineering.

For example, making a number of high-end suits, it should choose the fabric with a lot of wool fiber. Because wool fiber has good moisture absorption, good dyeing properties, flexibility, good thermal insulation, don't wrinkle and so on. After the dressing it, people looks very noble, elegant, solemn, generous, high quality of life. But in making underwear, according to the sanitary requirement of clothing ergonomics hygiene, wool fiber is not the best material of making underwear, cotton fiber is the best choice to make underwear, because the wool fiber failed to achieve the optimal conditions of the underwear should wash frequently, size stability, sanitary and soft, and it is easy to change color and mildew when it combines with the excreta of skin. And the cotton fiber is the most comfortable and soft fiber with a strong sweat absorption in all fibers, so it is recommended to be used as a material for underwear. Cotton fibers underwear has many advantages, but the only obvious drawback is the large shrinkage rate, it should be soaked before making clothing. But in general it is impossible that are soaked before the batch production, so the best way to solve the problem is using the blending material of cotton fiber and lycra elastic fiber to make underwear and using knitted stitch to weave, it can overcome the disadvantages of shrinkage of cotton fiber, and also ensures the stable size and shape preserving effect and has the good performance of sanitation, softness, compression, elasticity, absorbing sweat and ventilating.

Another example: Making sports wear and dancing dress should choose soft knitting material, because of the good performance of the stretching, compression, elasticity, sweat absorbent and breathable.

Another example: the Fordoo summer trousers is popular, because its fabric is silk and hemp fabric, using clothing ergonomics, aesthetics principle and other high-tech means to make clothing. And the hemp contains 26 kinds of trace elements and 10 kinds of amino acid that are beneficial to human health, the Fordoo summer trousers has the characteristics of strong heat dissipation, lightweight, comfortable, breathable, cool and so on, so the Fordoo summer trousers is favored by the large number of consumers, and bring rich economic benefits for the dealer.

\section{Clothing ERgonomics Is THE BASIS FOR THE NEW GeNeRATION OF Clothing Designers DEVELOPING to A HIGHER LEVEL}

Every clothing designer wants their clothing designed by themselves has the aesthetic feeling of the era and meet the needs of all people, but sometimes things will go contrary to people's wishes. The reason is that some designers focus on the beauty in form, ignoring the "people oriented", "humanized design", so the result can be imagined. Since the clothing engineering was published, in clothing design, designers more and more pay attention to meet people's requirements and the harmony and rapport of "human - clothing - environment", gradually change subjective expression of the beauty in form of the clothing design, the work reflects the rationalization and scientificity of design requirements. Clothing human body function has become the basis for this goal, people - clothing environment system constitutes a benign clothing behavior, the environment is the condition, clothing is the means, people are the focus.

Expect a new generation of designers has a open idea and the six concepts of modern clothing design, that is safe, healthy, comfortable, functional, aesthetic and personality. Because it is integration of system of the human and the human, the human and the clothing, the clothing and the environment, the environment and the human, is the new idea of clothing design. Therefore, designers must be based on the safety when they design clothing, focusing on pleasant; The clothing design must comply with the requirements of hygiene, make people keep away from damage after dressing; the designed clothing should make people calm, carefree, comfortable and beautiful, can not make the person feel cumbersome and can maximize play functional clothing, can enrich people's interest in life, and make people's spirit of the sentiment of sublimation.

Experts have said: "the six main ideas in the use of clothing design is the key to scientific, humane, and humanistic. Only when six concepts are arranged in a very harmonious suit, creative inspiration is consistent with the requirements of clothing ergonomics system, includes both clothing aesthetic meaning, but also has clothing rational content and function."You will become a talented and outstanding fashion designer, if you do like experts said.

\section{The DeVElopment Prospect OF Clothing Human ENGINEERING}

Ergonomics in China started late, in the early 1980s as a discipline established, as a branch of ergonomics -- clothing ergonomics is the beginning of the period at home, there are many designers and creative groups of clothing ergonomics don't know the meaning and research content objects, so it is not enough in terms of the clothing designing and processing, from the current situation, in order to change the design is simply the pursuit of formal beauty of thinking mode, still need to redouble our efforts and arduous task.

Human engineering, clothing ergonomics service for all design and creative activities, is the soul of all design industries. Along with the improvement of people's living standard, the appearance of the individual need, the change of 
the aesthetic interest, the design idea of the designer has also changed a lot. Design is designed for people, people is the starting point and destination of design. With the rapid development of scientific design, a lot of work that is directly manipulated by people will be replaced by computers. According to the relevant information records, "The 'GCAD' launched by the University of Geneva in Switzerland and the Swiss Federal Institute of technology, in computer via a threedimensional system for inspection of wearing clothing due to $3 \mathrm{D}$ graphics can rotate 360 degrees, so that people observed from multiple angles clothing styles in different replacement environment, different light source position, different color matching effect, the advanced design method and design concept indicates that clothing ergonomics of the future".

In fact, now the sophisticated technology research and development of clothing human body engineering is the core of the achievements of clothing brands. A series of new disciplines bring new research theories, methods and means for clothing ergonomics injected, also presents a series of research topics for the clothing of human engineering, greatly broaden the research scope and application range of clothing ergonomics, but also greatly promote the progress and development of clothing ergonomics.

\section{REFERENCES}

[1] Pan Jianhua. Garment Ergonomics Design. Shanghai. Donghua University Press, February 2015.

[2] Ni Yizhong. "Comfortability and Functionality of Garment Considered from Human Factors Engineering". Sichuan Silk, January 2006.

[3] Wang Pai. Human Engineering and Future, Journal of Environmental Management College of China. 2003.

[4] Xu Jun, Tao Kaishan. Human Engineering, Beijing: Chinese Textile Press, 2002. 